\title{
Determinação de propriedades de reservatórios através da análise de perfis geofísicos na Bacia de Taranaki- Nova Zelândia.
}

\author{
Ingra P. Martins Leal ${ }^{\star}$, UFF \\ Wagner Moreira Lupinacci, UFF
}

Copyright 2016, SBGf - Sociedade Brasileira de Geofísica

Este texto foi preparado para a apresentação no VII Simpósio Brasileiro de Geofísica, Ouro Preto, 25 a 27 de outubro de 2016. Seu conteúdo foi revisado pelo Comitê Técnico do VIISimBGf, mas não necessariamente representa a opinião da SBGf ou de seus associados. É proibida a reprodução total ou parcial deste material para propósitos comerciais sem prévia autorização da SBGf.

\begin{abstract}
Well logging is an important tool used in the oil and gas industry to measure properties of the geological environment. The information obtained from well logs contribute to depositional system and reservoirs characterization. In this paper, we used the interpretation of well logs to identify lithologies and to estimate the clay volume, porosity and saturation in two offshore wells of the New Zealand's Taranaki Basin. From these properties, we analyzed and concluded that the reservoirs of the Tui and Maui fields in this basin have many different characteristics with respect to the depositional system and the type of hydrocarbons, although they are close.
\end{abstract}

\section{Introdução}

Os perfis geofísicos têm como principais objetivos auxiliar em um melhor entendimento das camadas de subsuperfície e caracterizar propriedades de reservatórios. A partir de perfis de poços, podemos estimar propriedade das camadas como argilosidade, porosidade, permeabilidade, densidade e saturação. Com isto, muitas vezes conseguimos identificar e qualificar se o reservatório possui ou não condições de viabilidade econômica (Leite et al., 2007).

Em conjunto com a sísmica, a análise de perfis também gera uma maior segurança na determinação de melhores áreas para a perfuração, obtendo assim uma otimização de poços, aumentando o fator de recuperação e prolongando a vida útil do campo (Sacco et al., 2007).

Os reservatórios estudados neste trabalho estão localizados nos campos Tui e Maui na Bacia de TaranakiNova Zelândia (Figura 1). Para analisar estes reservatórios foram estimados as propriedades petrofísicas usando os dados dos poços Tui-1 e Maui-2 (as localizações destes poços são mostradas na Figura 2). O objetivo do trabalho foi estimar as propriedades de volume de argila, porosidade efetiva e saturação e fazer uma análise comparativa dos resultados nos campos analisados.
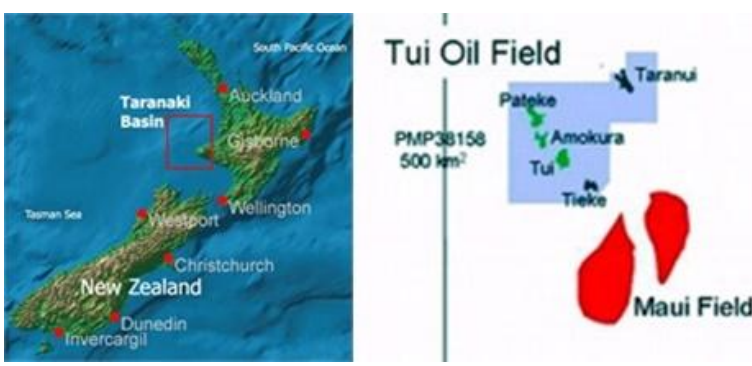

Figura 1: Localização dos campos Tui e Maui na Bacia de Taranaki. (Disponível em: http://www.rigzone.com/news/ oil gas/a/41045/Conversion Started on Maari FPSO V essel_Raroa. Acesso em: 14 de abril de 2016 .).

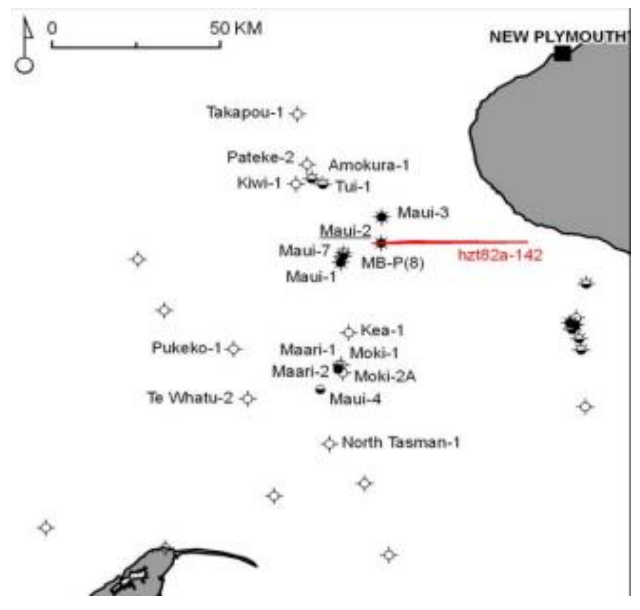

Figura 2: Localização dos poços "TUI-1" e "MAUI-2" (Disponível em: https://data.gns.cri.nz/pbe/\#HTML:Conte nt/outputs/Well\%20Sheets/wellsheets_2.html. Acesso em 27 de abril de 2016).

\section{Metodologia}

Os dados dos perfis de poços foram adquiridos no formato Comma Separated Values (CSV) disponibilizados no site do governo da Nova Zelândia. Os perfis necessários para o desenvolvimento da metodologia utilizados são: profundidade, raios gama (GR), sônico (DT), resistividade profunda (HLLD), densidade (RHOZ), nêutron (TNPH), azimute e desvio. E o fluxo de trabalho pode ser resumido nas seguintes etapas:

1) Levantamento bibliográfico sobre a área de interesse;

2) Aquisição dos dados digitais públicos dos poços Tui-1 e Maui-2; 


\section{Determinação de propriedades do reservatório através do método de perfilagem geofísica}

3) Importação e controle de qualidade dos dados para o software utilizado;

4) Processamento das curvas de desvios das profundidades dos poços para realização da computação da profundidade vertical verdadeira;

5) Criação da curva de temperatura de formação dos poços, com base no gradiente de temperatura de $40^{\circ} \mathrm{C} . \mathrm{Km}$. Dado adquirido no site do governo da Nova Zelândia;

6) Criação das zonas de análises usando o perfil GR;

7) Cálculo do volume de argila a partir do perfil GR usando a seguinte a equação :

$$
V_{\text {sh }}=\frac{G R_{\text {lido }}-G R_{\text {mínimo }}}{G R_{\text {máximo }}-G R_{\text {mínimo }}}
$$

no qual $V_{s h}$ é o volume de argila, $G R_{\text {lido }}$ o valor de raio gama medido pelo instrumento, $G R_{\text {mínimo }}$ a leitura do raio gama no arenito limpo, $G R_{\text {máximo }}$ a leitura do raio gama no folhelho.

8) Cálculo da porosidade a partir do método nêutrondensidade, que tem como base as equações 2, 3 e 4 . Para o perfil densidade temos:

$$
\phi_{d}=\frac{\rho_{\text {matriz }}-\rho_{\text {lido }}}{\rho_{\text {matriz }}-\rho_{\text {fluído }}}
$$

no qual $\phi_{d}$ é a porosidade calculada a partir do perfil densidade, $\rho_{\text {matriz }}$ a densidade da matriz, $\rho_{\text {lido }}$ a densidade lida pela ferramenta e $\rho_{\text {fluído }}$ a densidade do fluído. Para o perfil nêutron temos:

$$
\phi_{n}=\phi_{n e} S_{w} \phi_{n e}+\phi_{n e}\left(1-S_{w}\right) \phi_{n h}+V_{s h} \phi_{n s h}+\left(1-V_{s h}-\phi_{e}\right) \phi_{n i}
$$

no qual $\phi_{n}$ é a porosidade lida pela ferramenta, $\phi_{n e}$ a porosidade a ser estimada, $S_{w}$ o valor de saturação da água, $\phi_{n h}$ a leitura da ferramenta na zona de hidrocarboneto, $\phi_{n s h}$ a leitura na zona de folhelho, $V_{s h} \mathrm{o}$ volume de argila, $\phi_{n i}$ é a leitura no arenito. E por fim:

$$
\phi=\left(\frac{\phi_{d}^{2}+\phi_{n}^{2}}{2}\right)^{1 / 2}
$$

no qual $\phi$ é a porosidade calculada pelo método nêutrondensidade.

9) Cálculo da saturação de água usando o método de Archie:

$$
S_{w}^{n}=\frac{a R_{w}}{\phi^{m} R_{t}}
$$

no qual $\phi$ é a porosidade, $S_{w}$ a saturação de água, $a$ coeficiente de tortuosidade, $m$ o coeficiente de cimentação, $n$ o expoente de saturação, $R_{w}$ a resistividade da água e $R_{t}$ a resistividade da formação. Os valores das constantes usadas foram $a=1, m=2 \mathrm{e}$ $n=2$. Estes valores são normalmente utilizados na literatura para arenitos consolidados.

10) Cálculo da saturação de água pelo método de Simandoux:

$$
\left(\frac{\phi_{e}}{a R_{w}\left(1-V_{s h}\right)}\right) S_{w}^{n}+\frac{V_{s h}}{R_{s h}} S_{w}=\frac{1}{R_{t}}
$$

no qual $R_{s h}$ a resistividade da argila.

11) Estimativa da saturação de hidrocarboneto, $S_{h}$, na zona do reservatório:

$$
S_{h}=1-S_{w}
$$

12) Interpretação dos resultados obtidos.

\section{Resultados e Discussões}

Os resultados foram obtidos e interpretados após a aplicação do fluxo mostrado na seção da metodologia. Os valores utilizados para os parâmetros $G R_{\text {minimo }}$, $G R_{\text {máximo }}, \rho_{\text {matriz }}, \rho_{\text {fluído }}, S_{w}, R_{w}$ e $R_{t}$ foram obtidos através de análise gráfica ou interpretação dos perfis (Contreras e Castro, 2012).

A área de interesse do poço de Tui-1 foi fixada entre $3657 \mathrm{~m}$ e $3683 \mathrm{~m}$ que é mostrada na figura 3. No poço Maui-2, a área de interesse foi entre $2721 \mathrm{~m}$ e $2874 \mathrm{~m}$, visualizada na figura 4 . Nestes poços, estas áreas foram determinadas a partir da análise dos perfis de resistividade, nêutron e densidade.

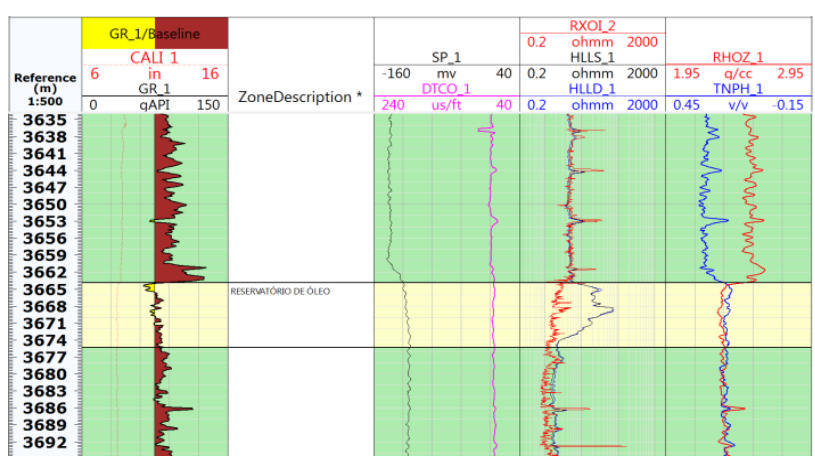

Figura 3: Perfis do poço Tui-1 destacando a zona de interesse.

Os perfis calculados de volume de argila, porosidade e saturação para os poços Tui-1 e Maui-2 são mostrados nas figuras 5 e 6 , respectivamente. Os valores médios dos volumes de argilas encontrados foram $14,9 \%$ para o poço Tui-1 e 6\% para o poço Maui-2. O valor moderado do volume de argila obtido no poço Tui-1 mostra que o reservatório trata-se de um arenito argiloso. Isto impacta diretamente na porosidade e permeabilidade, pois quanto maior for o volume de argila menor tente a ser a 
porosidade efetiva e a permeabilidade, o que gera dificuldades para o escoamento do óleo.

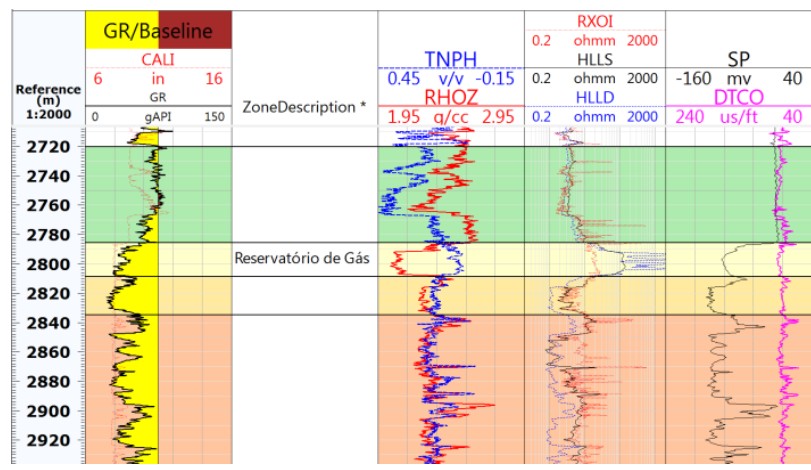

Figura 4: Perfis do poço "Maui-2" destacando a zona de interesse.

Com base na análise e padrão de acomodação do volume argila em cada reservatório é possível inferir que na formação Tui ocorreu um hiato deposicional com uma mudança abrupta de granulometria na zona do reservatório mostrando um padrão granulocrescente que é típico de um ambiente transgressivo. Já na formação Maui trata-se de um reservatório de arenito limpo com uma mudança gradativa na granulometria com um padrão granulodecrescente, típico de um ambiente regressivo, seguido de um hiato deposicional.

Usando os perfis de resistividades foram encontrados uma zona de óleo entre $3664 \mathrm{~m}$ e $3675 \mathrm{~m}$ no poço Tui-1 e uma zona de gás entre $2785 \mathrm{~m}$ a $2808 \mathrm{~m}$ e uma zona de óleo entre $2808 \mathrm{~m}$ e $2835 \mathrm{~m}$ no poço Maui-2. Os valores de resistividade no poço Maui-2 não foram menores $2 \Omega \mathrm{m}$, devido se tratar de um reservatório de gás. Isto também pode indicar uma baixa saturação de água. No poço Tui-1, os valores de resistividade quando comparado com o poço Maui-2 são menores por se tratar de um reservatório de óleo e ele também mostrou uma maior saturação de água no intervalo de interesse do mesmo.

Nos utilizamos o crossover entre os perfis densidade e nêutron para identificar a zona de reservatório de gás no poço Maui-2. Para esta análise, sabemos que ocorre diminuição da densidade e um aumento da porosidade nêutron na presença de uma zona de gás (Nery, 2013).

Os valores médios das porosidades obtidas usando o método de nêutron-densidade foram de $16,7 \%$ no poço Tui-1 e 23,5\% no poço Maui-2. Com estes valores foi possível estimar a saturação de água nos reservatórios. O valor obtido para o poço Tui-1 foi de $31,9 \%$ pelo Método de Archie e de $31,0 \%$ pelo método de Simandoux. Para o poço Maui-2, os valores de saturação de água obtidos foram de $14,1 \%$ pelo método de Archie e 10,4\% pelo método de Simandoux. Essa diferença se deve ao fato de que o método de Archie superestima saturações de água nos intervalos argilosos. Por isto, este método é comumente utilizado em reservatórios com baixa concentração de argila. Já o método de Simandoux é utilizado para corrigir o efeito da presença de argila nos sedimentos. No poço Maui-2, apesar do reservatório ter um volume de argila pequeno, possui uma porosidade média alta, o que contribuiu para uma maior diferença do valor de saturação encontrado entre os métodos de Archie e Simandoux.

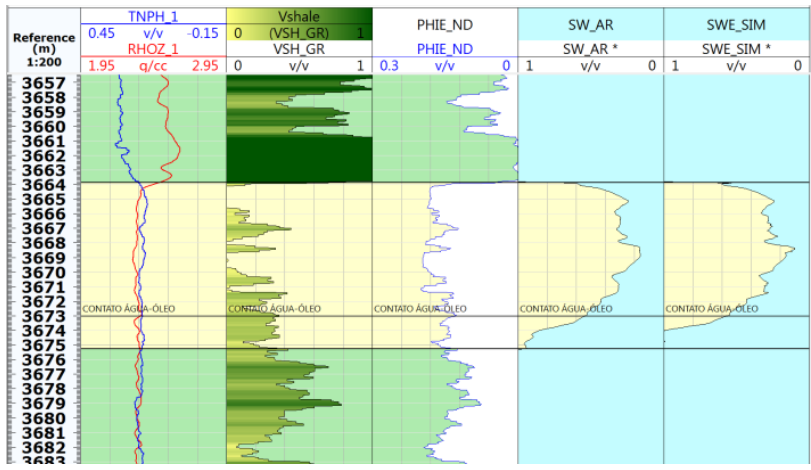

Figura 5: Perfis de densidade e de nêutron e perfis calculados de volume de argila, porosidade efetiva, saturação pelo método de Archie e saturação pelo método de simandoux para o poço de Tui-1.

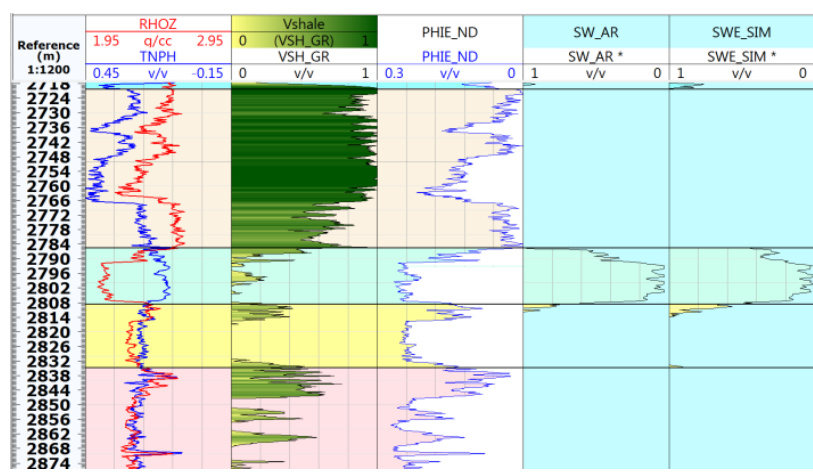

Figura 6: Perfis de densidade e de nêutron e perfis calculados de volume de argila, porosidade efetiva, saturação pelo método de Archie e saturação pelo método de Simandoux para o poço de "MAUI-2".

\section{Conclusões}

Com o uso das técnicas de interpretação de perfis de poços foi possível estimar e analisar as propriedades dos reservatórios nos campos Tui e Maui. No poço Tui-1 constatou que se trata de um reservatório de óleo, com moderada presença de argila e uma mudança abrupta de granulometria na zona do reservatório com um padrão granulocrescente típico de um ambiente transgressivo. Já no poço MAUI-2 trata-se de um reservatório de gás com baixo conteúdo de argila e uma mudança gradativa na granulometria com um padrão granulodecrescente típico de um ambiente regressivo seguido de um hiato deposicional. Com este estudo, nos podemos perceber que apesar dos campos Tui e Maui estarem próximos e pertencerem a mesma Bacia Sedimentar (Bacia de Taranaki), os reservatórios apresentam características bem distintas.

\section{Agradecimentos}

Os autores agradecem ao Sr. Nuno André Pomar Couto que contribuiu para a preparação deste trabalho. 


\section{Referências}

Contreras, Sérgio A. C., Castro, J. C., 2012. Metodologia para determinar parâmetros petrofísicos de corte em um campo de petróleo: o caso Socororo, bacia do Oriente, Venezuela. Revista Escola de Minas, v. 65, n 3, 305312.

Leite, M., Carrasquilla, A, Silva, J., Lobo, L., Curtinhas, R.; Batista, S., 2007. Aplicação da perfilagem geofísica em projetos de perfuração de poços de petróleo. 10th International Congress of the Brazilian Geophysical Society.

Nery, G. G., 2013. Perfilagem geofísica em poço aberto fundamentos básicos com ênfase em petróleo. INCTGP/CNPq - SBGf.

Sacco, T.; Suslick, S. B.; Vidal, A. C., 2007. Modelagem Geológica 3D do Campo de Namorado Utilizando Dados de Perfilagem de Poços Verticais. $4^{\circ}$ PDPETRO. 